Сравнение вариантов анестезии и периоперационной анальгезии при симультанной гернио-и абдоминопластике вентральных грыж у пациентов с ожирением

\author{
М.И. Неймарк, Р.В. Киселев
}

ГБОУ ВПО «Алтайский государственный медицинский университет» МЗ РФ, Барнаул

Обоснование. Среди пациентов с большими послеоперационными вентральными грыжами нередко встречаются больные с высокой степенью ожирения. Эта группа пациентов представляет определенную сложность при проведении анестезиологического обеспечения в связи с целым рядом анатомо-физиологических изменений и высоким риском развития осложнений в раннем послеоперационном периоде, что явилось основанием для выполнения настоящего исследования.

Цель исследования. Изучение влияния различных вариантов анестезиологического пособия и периоперационной анальгезии при симультанной гернио- и абдоминопластики больших послеоперационных вентральных грыж (ПВГ) у пациентов с сопутствующим ожирением на течение периоперационного периода.

Материалы и методы. Проведено рандомизированное исследование 59 пациентов с индексом массы тела $>30$ кг/м². В зависимости от вида анестезиологического пособия пациенты поделены на две группы. В 1-й группе $(n=30)$ операция выполнена в условиях сочетанной анестезии на основе низкопоточной (low flow) ингаляции десфлурана в комбинации с продленной эпидуральной анальгезией (ПЭА) ропивакаином, во 2-й группе $(n=29)$ операция выполнена в условиях сочетанной анестезии на основе low flow ингаляции десфлурана в комбинации с блокадой поперечного пространства живота (transversus abdominal plane - TAP) 0,5\% раствором ропивакаина. Исследовались показатели адекватности анестезии, центральной и периферической гемодинамики, проводился мониторинг нейромышечной проводимости, оценивались эффективность послеоперационной реабилитации и качество послеоперационной анальгезии, количество послеоперационных критических инцидентов.

Результаты. Было выявлено, что оперативное вмешательство в условиях низкопоточной ингаляционной анестезии на основе десфлурана в сочетании в ПЭА ропивакаином способствует более быстрой постнаркозной реабилитации, эффективной послеоперационной анальгезии и меньшему количеству осложнений в раннем

\section{Comparison of anesthesia and perioperative analgesia in simulty hernioplasty and abdominoplasty in patients with obesity}

\author{
M.I. Neimark, R.V. Kiselev \\ Altai State Medical University of the Ministry of Health of Russia, \\ Barnaul
}

Justification. Among patients with large postoperative ventral hernias, the most common are patients with varying degrees of obesity. This group of patients presents a certain complexity for anesthesia in connection with a number of anatomical and physiological changes and a high risk of complications in the early postoperative period in connection with which this study was conducted.

Purpose of trial. Study of the influence of various anaesthetic management and perioperative analgesia with simultaneous hernioplasty and abdominoplasty of major postoperative incisional hernia $(\mathrm{PIH})$ in patients with obesity during the perioperative period.

Materials and methods. A randomized trial of 59 patients with a body mass index $>30 \mathrm{~kg} / \mathrm{m}^{2}$ was performed. Depending on the type of anesthesia, patients are divided into two groups. In the $1^{\text {st }}$ group $(n=30)$, the operation was performed on low-flow inhalation of desflurane based anaesthesia in combination with prolonged epidural analgesia (PEA) with ropivacaine, in the $2^{\text {nd }}$ group $(n=29)$, the operation was performed on low flow inhalation of desflurane anesthesia based in combination with blockade of transverse abdominal plane (TAP) with $0.5 \%$ solution of ropivacaine. The indicators adequacy of anesthesia parameters investigated, central and peripheral hemodynamics were monitored neuromuscular conduction, evaluated the efficacy of recovery after surgery and postoperative analgesia quality, postoperative critical incident were studied.

Results. It was found that surgical intervention in lowflow inhalation desflurane anaesthesia based combined with PEA ropivacaine promotes faster recovery after surgery and effective postoperative analgesia and fewer complications in the early postoperative period, which contributed to significantly shorter hospital lengths of stay for patients in group $1-82,5$ hours $(95 \% \mathrm{Cl} 76-93,5)$ compared with group $2-94$ hours $(95 \% \mathrm{Cl} 85,5-113)$ $(p=0,015)$.

Conclusions. Combined desflurane-based anesthesia in combination with regional methods is an effective method of anesthesia and analgesia in the perioperative period 
послеоперационном периоде по сравнению с использованием блокады ТАР. Это способствовало достоверно меньшим срокам госпитализации пациентов в 1-й группе - 82,5 ч (95\% ДИ 76-93,5) в сравнении со 2-й группой - 94 ч (95\% ДИ 85,5-113) ( $(=0,015)$.

Выводы. Сочетанная анестезия на основе десфлурана в комбинации с ПЭА является эффективным методом анестезии и анальгезии в периоперационном периоде, способствует более быстрой постнаркозной реабилитации и сокращению сроков госпитализации при симультанной гернио- и абдоминопластике больших ПВГ у пациентов с ожирением по сравнению с блокадой ТАР.

\section{Ключевые слова:}

низкопоточная анестезия, продленная эпидуральная анальгезия, послеоперационная анальгезия, ожирение, герниопластика, блокада поперечного пространства живота

凶 Для корреспонденции: Киселев Роман Владимирович, канд. мед. наук, ассистент кафедры анестезиологии, реаниматологии, клинической фармакологии с курсом ДПО ГБОУ ВПО «Алтайский государственный медицинский университет» Минздрава России, Барнаул; e-mail: fincher-75@mail.ru

匹 Для цитирования: Неймарк М.И., Киселев Р.В. Сравнение вариантов анестезии и периоперационной анальгезии при симультанной гернио- и абдоминопластике вентральных грыж у пациентов с ожирением. Вестник интенсивной терапии имени А.И. Салтанова. 2019;1:45-51. and contributes to faster recovery after surgery and shorter surgical treatment with simultaneous hernio- and abdominoplasty of large (PIH) in obese patients.

\section{Keywords:}

low-flow anesthesia; prolonged epidural analgesia, postoperative analgesia, obesity, hernioplasty, blockage of the transverse abdominal plane

\ For correspondence: Roman V. Kiselev, Ph.D., Assistant of the Department of Anaesthesiology, Resustitation, Clinical Pharmacology with the course of postgraduate education of the Altai State Medical University of the Ministry of Health of the Russian Federation, Barnaul; e-mail: fincher-75@mail.ru

๘ For citation: Neimark MI, Kiselev RV. Comparison of anesthesia and perioperative analgesia in simulty hernioplasty and abdominoplasty in patients with obesity. Alexander Saltanov Intensive Care Herald. 2019;1:45-51.

\section{Введение}

Лечение больных с грыжами передней брюшной стенки остается актуальной проблемой хирургии. Это обусловлено тем, что число послеоперационных грыж в структуре наружных грыж живота постоянно растет, достигая в настоящее время 20 \% всех операций на органах брюшной полости $[1,2]$. Это связано с ростом числа травматичных и продолжительных операций в абдоминальной хирургии, в том числе этапных и повторных вмешательств, а также постоянным увеличением среди оперируемых лиц с сопутствующим ожирением. При этом ожирение само по себе является одной из причин возникновения послеоперационных вентральных грыж (ПВГ) $[3,4]$. Пациенты, имеющие ожирение различной степени, составляют до 64 \% всех больных с ПВГ [5, 6]. Как правило, такой контингент больных представляет определенную сложность для анестезиологического обеспечения хирургического лечения в связи с имеющимися серьезными анатомо-физиологическими изменениями, вероятными техническими трудностями на всех этапах анестезии, а также высоким риском развития осложнений в послеоперационном периоде.

Цель исследования: изучение влияния различных вариантов анестезиологического пособия и периоперационной анальгезии при симультанной гернио- и абдоминопластике больших ПВГ у пациентов с ожирением на течение периоперационного периода.

\section{Материалы и методы исследования}

Проведено рандомизированное проспективное исследование 59 пациентов с индексом массы тела (ИМТ) $>30$ кг $/$ м $^{2}$, которым была выполнена симультанная гернио- и абдоминопластика большой послеоперационной вентральной грыжи в хирургической клинике Отделенческой клинической больницы на станции Барнаул ОАО «РЖД». В зависимости от выбора анестезиологического пособия пациенты были разделены на две группы. В 1-й группе $(n=30)$ операция выполнена в ус- 
ловиях сочетанной анестезии на основе low flow ингаляции десфлурана в комбинации с продленной эпидуральной анальгезией (ПЭА) 0,2\% раствором ропивакаина, во 2-й группе $(n=29)$ операция выполнена в условиях сочетанной анестезии на основе low flow ингаляции десфлурана в комбинации с блокадой поперечного пространства живота (transversus abdominal plane - TAP) 0,5\% раствором ропивакаина [7]. По 6 основным признакам сравниваемые группы были репрезентативны: пол, возраст, ИМТ, характер сопутствующей патологии, физическое состояние по ASA, тип оперативного вмешательства. Критерии исключения: возраст < 15 лет и > 75 лет, ИМТ < 30, хроническая сердечная недостаточность по классификации NYHA - II функциональный класс и выше, хроническая дыхательная недостаточность - II степени и выше, уровень гемоглобина $<120$ г/л для мужчин и < 110 г/л - для женщин.

Всем пациентам вечером для профилактики тромбоэмболических осложнений за 12 ч до операции вводились подкожно низкомолекулярные гепарины (эноксапарин 80 мг). Для профилактики развития стресс-индуцированного повреждения ЖКТ внутривенно вводили ингибиторы протонной помпы (эзомепразол 40 мг) за 1 ч до индукции в анестезию. Антибиотикопрофилактика достигалась введением амоксициллина/клавуланата 1200 мг в/за 2 ч. В 1-й группе в операционной с УЗИ-ассистированием катетеризировали эпидуральное пространство на уровне Th10-Th11. Катетер 20G проводили краниально на 3 см и фиксировали стерильными наборами, далее вводили тест-дозу местного анестетика (ропивакаин $0,5 \% 2,0)$. Через 5 минут (при отсутствии признаков спинальной анестезии) начинали пошаговое введение 0,2\% ропивакаина болюсами по 3,0 мл до введения полной дозы 11,5 \pm 1,5 мл в течение 20-30 минут. Через 1,52,0 ч начинали инфузию поддерживающей дозы $0,2 \%$ ропивакаина со скоростью 8,0 \pm 1,0 мл/час. Индукцию в анестезию проводили фентанилом 2,5 \pm 0,07 мкг/кг тощей массы тела и пропофолом 2,5 \pm 0,03 мг/кг актуальной массы тела. Интубацию трахеи выполняли на фоне миорелаксации рокурония бромидом 0,6 \pm 0,04 мг/кг идеальной массы тела. Базовая анестезия поддерживалась low flow ингаляцией десфлурана в дозе в сочетании с ПЭА ропивакаином со скоростью 5-8 мл/ч. Миорелаксацию поддерживали рокурония бромидом $-0,1$ мг/кг/ч тощей массы тела. Во 2-й группе индукция в анестезию осуществлялась аналогично с 1-й группой, после индукции в условиях УЗИ-ассистирования с использованием игл для проводниковой анестезии с эхогенными метками осуществлялась двусторонняя блокада поперечного пространства живота введением 0,5\% раствора ропивакаина в фасциальное пространство поперечной мышцы живота - по 30 мл на каждую сторону. Базовая анестезия поддерживалась low flow ингаляцией десфлурана, а миорелаксация - внутривенной инфузией рокурония бромида. Интраоперационная инфузионная терапия в группах проводилась с учетом патологических и физио- логических потерь сбалансированными солевыми растворами. Интраоперационная искусственная вентиляция легких - в режиме $\mathrm{PCV}+\mathrm{PEEP}$.

Послеоперационную анальгезию у пациентов 1-й группы осуществляли мультимодальным методом по следующей схеме: в эпидуральное пространство перфузором вводился 0,2\% раствор ропивакаина со скоростью 7-12 мл/ч, эпидуральная анальгезия комбинировалась болюсным введением нестероидных противовоспалительных препаратов (НПВП) - кеторолак 90 мг/сут, а также инфузией парацетамола - перфалган 4 г/сут.

При неэффективности анальгезии дополнительно вводился болюс промедола - 20 мг в/м. В послеоперационном периоде у пациентов 2-й группы ТАР блок комбинировался с НПВП - кеторолак 90 мг/сут, и парацетамолом - перфалган 4 г/сут; при неэффективности анальгезия потенцировалась опиоидами - болюс промедол 20 мг в/м. Исследования проводили на 4 этапах: непосредственно перед индукцией в анестезию, на этапе пластики грыжевых ворот, удаления избыточных кожно-жировых лоскутов при абдоминопластике, послойного ушивания раны. Глубину анестезии мониторировали с помощью биспектрального индекса модулем BISX ${ }^{\mathrm{TM}}$ (Coviden, USA), поддерживая показатель BIS на уровне 50-60 [8]. Адекватность анестезии оценивали по уровню диастолического артериального давления (ДАД), артериовенозная разница по кислороду (a-vDO$)$, сдвиг буферных оснований (BE), насыщение смешанной венозной крови кислородом $\left(\mathrm{S}_{\mathrm{v}} \mathrm{O}_{2}\right)$, минутному диурезу, индексу BIS [9]. Нейромышечный мониторинг выполняли методом акселеромиографии с помощью системы TOF-Watch ${ }^{\odot}$ SX (Organon, Ireland), не допуская более одного ответа в режиме TOF-стимуляции. Фармакологическую реверсию нейромышечного блока осуществляли при появлении Т2 в режиме ТOF-стимуляции сугаммадексом в дозе 2 мг/кг. Экстубацию трахеи осуществляли при достижении индекса TOF 0,9 и клинических признаков ее восстановления: способность поднятия и удержания головы над операционным столом в течение 5 с (тест Дама), сила рукопожатия.

Эффективность и качество постнаркозной реабилитации оценивали по времени достижения больными индекса BIS > 95 и экстубации пациентов, времени достижения 10 баллов по шкале пробуждения Aldrete, 0 баллов по тесту исчезновения послеоперационной сонливости и восстановления ориентированности Bidway, первого вставания на ноги, восстановления перистальтики, отхождения газов, продолжительности пребывания в стационаре. Эффективность анальгезии оценивали с помощью 100-миллиметровой визуально-аналоговой шкалы (ВАШ), по времени первого требования анальгетика, расходу наркотического анальгетика в послеоперационном периоде. Для исключения аггравации пациентами выраженности болевого синдрома из-за опасения снижения дозы анальгетика, так называемый симптом стра- 
ха повторной боли, цифровые значения ВАШ дублировались словесными характеристиками интенсивности боли по 10-балльной вербальной описательной шкале оценки боли Verbal Descriptor Scale [10]. В послеоперационном периоде регистрировали частоту критических инцидентов (гипоксемия $\mathrm{SpO}_{2}<90 \%$, бронхоспазм, парез кишечника, послеоперационная тошнота и рвота, задержка мочеиспускания, нарушения сердечного ритма, тромботические осложнения: тромбоэмболия легочной артерии и тромбоз глубоких вен) $[11,12]$.

\section{Статистическая обработка}

Количественные переменные проанализированы на нормальность распределения с помощью теста Шапиро-Уилка и Лиллиефорса. В том случае, если распределение соответствовало нормальному, для оценки достоверности различий между выборками использовался t-критерий Стьюдента. В противном случае использовался U-критерий Манна-Уитни. Для сравнения категориальных переменных использовался $\chi^{2}$-тест Пирсона (с поправкой Йетса при анализе таблиц сопряженности типа $2 \times 2$, т.е. при степени $=1)$. Для анализа динамики с нормальным распределением использовался t-критерий Стьюдента для связанных выборок, в случае с ненормальным распределением применялся Т-критерий Вилкоксона. Уровень статистической значимости при проверке нулевой гипотезы принимали соответствующий $p<0,05$. Обработку данных проводили с помощью пакета программ SPSS Statistics 19.0.

Локальный этический комитет Алтайского государственного университета одобрил исследование «Сравнение вариантов анестезии и периоперационной анальгезии при симультанной гернио- и абдоменопластике больших послеоперационных вентральных грыж у пациентов с ожирением».

\section{Результаты и их обсуждение}

Средняя продолжительность оперативного вмешательства в группах, суммарный объем интраоперационной кровопотери и потери по дренажам, а также объем инфузионной терапии достоверно между группами не различались.

В ходе анализа критериев адекватности анестезии в группах начиная со 2-го этапа исследования и на последующих этапах зарегистрирован достоверно более низкий уровень ДАД в 1-й и 2-й группах в сравнении с исходным этапом исследования. Кроме того, выявлено достоверное увеличение показателей $\mathrm{Sv}_{2}, \mathrm{a}-\mathrm{vDO}_{2}$ в обеих группах в сравнении с 1-м этапом исследования. На дальнейших этапах исследования различий между группами в этих показателях выявлено не было, однако зарегистрировано различие в темпе диуреза, который у пациентов 1-й группы был достоверно выше начиная со 2-го и на последующих этапах в сравнении со 2-й группой (табл. 1).

При исследовании эффективности постнаркозной реабилитации не было зарегистрировано достоверного различия во времени достижения индекса BIS > 95 после окончания операции между пациентами 1-й группы $12(8,5-19,5)$ мин и 2-й группы - $11(7,5-18)(p=0,125)$, времени экстубации в 1-й группе - $14(8,5-18)$ мин, во 2-й группе - 14 (9,5-19) ( $p=0,089)$, времени пробуждения и достижения 10 баллов по шкале Aldrete у больных 1-й группы - $3(2,5-6,5)$ мин, 2-й группы 3,5 (3-7) мин $(p=0,231)$. Время достижения 0 баллов по тесту Bidway у больных 1-й группы - 4 (2,5-7) мин, 2 -й группы - 4,5 $(2,5-7,5)$ мин $(p=0,076)$. Первый подъем на ноги в 1-й группе произошел достоверно раньше - 186 (135-226) мин в сравнении со 2-й группой -213 (144-258) мин $(p=0,033)$. Первые шумы перистальтики кишечника у пациентов 1-й группы появились достоверно раньше, чем у пациентов 2-й группы - 207 (175-232) и 354 (305-441) мин соответственно $(p=0,043)$, также зарегистрировано более раннее начало отхождения газов после операции в 1-й группе - 514,8 $(481,7-555,1)$ мин в сравнении со 2-й группой $-596,1(537,1-623,2)$ мин $(p=0,039)$. Кроме того, время пребывания в стационаре у пациентов 1-й группы - 27,3 $(18,4-31,3)$ ч было достоверно меньшим, чем у пациентов 2-й группы - 42,5 $(37,8 ; 51,9)$ ч $(p=0,032)$.

При сравнении интенсивности болевых ощущений по ВАШ в первые сутки после операции достоверного различия между группами выявлено не было. Так, у пациентов 1-й группы интенсивность болевых ощущений через 1 час после операции составила 34 (29- 43) мм, во 2-й группе - 35 (29-44) мм $(p=0,085)$, через 6 ч после операции в 1-й группе - $33(30-40)$ мм, во 2-й группе $-34(31-41)$ мм $(p=0,076)$, через 12 ч в 1-й группе 32 (26-37) мм, во 2-й группе - $33(29-37)$ мм $(p=0,127)$, однако через 24 ч в 1-й группе интенсивность болевого синдрома была достоверно ниже - 20 (15-25) мм, во 2-й группе $-31(24-35)$ мм $(p=0,045)$. Значения интенсивности болевого синдрома по шкале Verbal Descriptor Scale в группах также имели схожую динамику. В первые сутки после операции достоверного различия не было. Так, у пациентов 1-й группы интенсивность болевого синдрома через 1 час после операции составила 2,5 (2-3,5) балла, у пациентов 2-й группы $3(2,5-3,5)$ балла $(p=0,137)$, через 6 ч после операции у пациентов 1-й группы - 2 (2-3) балла, у пациентов 2-й группы - 2,5 $(2,5-3,5)$ балла $(p=0,076)$, через 12 ч у пациентов 1 -й группы - $2(1,5-2,5)$ балла, у пациентов 2-й группы - $2(1,5-3)$ балла $(p=0,069)$, через 24 ч у пациентов 1-й группы интенсивность болевого синдрома была достоверно ниже - 1,5 (0,6-1,5), 2-й группы $-2,5(2-3)$ ( $p=0,042)$. Соответственно, при отсутствии различия в интенсивности болевого синдрома в группах в первые сутки после операции не было выявлено достоверного различия во времени первого 


\begin{tabular}{|c|c|c|c|c|c|}
\hline \multirow{2}{*}{$\begin{array}{c}\text { Исследуемые } \\
\text { показатели }\end{array}$} & \multirow{2}{*}{$\begin{array}{l}\text { Исследуемые } \\
\text { группы }\end{array}$} & \multicolumn{4}{|c|}{ Этапы исследования } \\
\hline & & 1-й этап & 2-й этап & 3-й этап & 4-й этап \\
\hline \multirow[t]{2}{*}{$\begin{array}{l}\text { Медиана (МКИ) } \\
a-v D O_{2}, \text { мл/дл }\end{array}$} & 1-я группа & $2,4(1,5-2,9)$ & $\begin{array}{c}9,1(8,7-9,6) \\
P_{1}=0,012\end{array}$ & $\begin{array}{c}9,4(8,8-9,7) \\
P_{1}=0,005\end{array}$ & $9,7(8,8-9,7) \boldsymbol{P}_{1}=0,015$ \\
\hline & 2-я группа & $\begin{array}{c}2,9(1,8-3,2) \\
P_{2}=0,166\end{array}$ & $\begin{array}{c}9,8(9,2-10,3) \\
P_{1}=0,009 \\
P_{2}=0,167\end{array}$ & $\begin{array}{c}9,5(8,7-10,1) \\
P_{1}=0,009 \\
P_{2}=0,364\end{array}$ & $\begin{array}{c}9,6(8,7-9,9) p_{1}=0,013 \\
P_{2}=0,306\end{array}$ \\
\hline \multirow[t]{2}{*}{$\begin{array}{l}\text { Медиана (МКИ) } \\
\text { ВЕ, ммоль/л }\end{array}$} & 1-я группа & $-1,1(-1,9 \ldots+1,2)$ & $\begin{array}{c}-1,4(-1,1 \ldots+1,6) \\
P_{1}=0,087\end{array}$ & $\begin{array}{c}-1,3(-0,8 \ldots+1,4) \\
P_{1}=0,211\end{array}$ & $\begin{array}{c}-1,4(-0,9 \ldots+1,4) \\
P_{1}=0,087\end{array}$ \\
\hline & 2-я группа & $\begin{array}{c}-1,2(-2,0 \ldots+1,6) \\
P_{2}=0,165\end{array}$ & $\begin{array}{c}-1,3(-1,1 \ldots+1,5) \\
P_{1}=0,096 \\
P_{2}=0,122\end{array}$ & $\begin{array}{c}-1,4(-1,2 \ldots+1,5) \\
P_{1}=0,269 \\
P_{2}=0,184\end{array}$ & $\begin{array}{c}-1,2(-0,8 \ldots+1,3) \\
P_{1}=0,238 \\
P_{2}=0,145\end{array}$ \\
\hline \multirow[t]{2}{*}{$\begin{array}{l}\text { Диурез, среднее } \\
\pm \text { SD, мл/мин }\end{array}$} & 1-я группа & $47,2 \pm 2,7$ & $\begin{array}{l}58,4 \pm 2,9 \\
p_{1}=0,045\end{array}$ & $\begin{array}{l}59,5 \pm 2,8 \\
P_{1}=0,039\end{array}$ & $\begin{array}{l}58,9 \pm 2,9 \\
P_{1}=0,048\end{array}$ \\
\hline & 2-я группа & $\begin{array}{l}48,2 \pm 3,2 \\
P_{2}=0,157\end{array}$ & $\begin{array}{l}48,1 \pm 3,5 \\
P_{1}=0,234 \\
P_{2}=0,042\end{array}$ & $\begin{array}{l}49,3 \pm 3,2 \\
P_{1}=0,267 \\
P_{2}=0,039\end{array}$ & $\begin{array}{l}48,6 \pm 3,3 \\
P_{1}=0,234 \\
P_{2}=0,022\end{array}$ \\
\hline \multirow[t]{2}{*}{$\begin{array}{l}\text { ДАД, среднее } \\
\pm \text { SD, мм рт. ст. }\end{array}$} & 1-я группа & $88,3 \pm 2,8$ & $\begin{array}{l}71,3 \pm 2,8 \\
P_{1}=0,036\end{array}$ & $\begin{array}{l}72,6 \pm 2,1 \\
P_{1}=0,041\end{array}$ & $\begin{array}{l}72,9 \pm 2,5 \\
p_{1}=0,028\end{array}$ \\
\hline & 2-я группа & $\begin{array}{l}91,6 \pm 2,4 \\
P_{2}=0,087\end{array}$ & $\begin{array}{l}72,5 \pm 2,8 \\
P_{1}=0,027 \\
P_{2}=0,071\end{array}$ & $\begin{array}{l}73,3 \pm 2,8 \\
P_{1}=0,026 \\
P_{2}=0,117\end{array}$ & $\begin{array}{l}72,5 \pm 2,9 \\
P_{1}=0,034 \\
P_{2}=0,201\end{array}$ \\
\hline \multirow[t]{2}{*}{$\begin{array}{l}\mathrm{S}_{\tilde{V}} \mathrm{O}_{2}, \text { среднее } \\
\pm \mathrm{SD}, \%\end{array}$} & 1-я группа & $71,3 \pm 2,6$ & $\begin{array}{l}79,3 \pm 2,3 \\
p_{1}=0,036\end{array}$ & $\begin{array}{l}80,6 \pm 2,4 \\
P_{1}=0,041\end{array}$ & $\begin{array}{l}79,9 \pm 2,5 \\
P_{1}=0,027\end{array}$ \\
\hline & 2-я группа & $\begin{array}{l}72,6 \pm 3,1 \\
P_{2}=0,134\end{array}$ & $\begin{array}{l}77,5 \pm 3,2 \\
P_{1}=0,032 \\
P_{2}=0,072\end{array}$ & $\begin{array}{l}79,3 \pm 3,3 \\
P_{1}=0,035 \\
P_{2}=0,189\end{array}$ & $\begin{array}{l}80,5 \pm 3,1 \\
P_{1}=0,034 \\
P_{2}=0,082\end{array}$ \\
\hline
\end{tabular}

a-vDO 2 - артериовенозная разница по кислороду; ВE - сдвиг буферных оснований; SD - стандартное отклонение; Sर̃O 2 - насыщение смешанной венозной крови кислородом; ДАД - диастолическое артериальное давление; МКИ - межквартильный интервал. $p_{1}$ - достоверность различия в группах по сравнению с исходным этапом; $p_{2}-$ достоверность различия между 1-й и 2-й группой; жирным шрифтом выделена разница между группами при $p<0,05$.

требования анальгетика между группами: в 1-й группе -59 (54-63) мин и во 2-й группе -58 (52-63) мин $(p=0,075)$. При подсчете суммарного расхода наркотических анальгетиков в первые сутки расход промедола в группах достоверно не различался. В 1-й группе составил $21(17-25)$ мг, во 2-й группе $-22(18-28)$ мг $(p=0,083)$, на 2-е сутки суммарный расход промедола в 1-й группе был достоверно меньшим $-12(9,5-18)$ мг, чем во 2-й группе $-24(18,5-29)$ мг ( $p=0,036)$.

В раннем послеоперационном периоде в 1-й группе было зарегистрировано достоверно меньшее количество критических инцидентов в сравнении со 2-й группой (табл. 2), а также зарегистрированы достоверно меньшие сроки госпитализации пациентов в 1-й группе $-82,5$ ч (95\% ДИ 76-93,5) в сравнении со 2-й группой 94 ч $(95 \%$ ДИ 85,5-113) $(p=0,015)$.

\section{Обсуждение}

При одинаковых показателях объема инфузионной терапии кровопотери и глубины анестезии по BIS и других критериев адекватности анестезии обнаружено увеличение диуреза в 1-й группе, что связано с улучшением почечного и спланхнического кровотока на фоне эпидуральной симпатической блокады. Увеличение значений показателей содержания кислорода в смешанной венозной крови $\left(\mathrm{C} \tilde{\mathrm{v}} \mathrm{O}_{2}\right), \mathrm{a}-\mathrm{vDO} \mathrm{D}_{2}$ в обеих группах в сравнении с начальным этапом исследования связанно с адекватной интраоперационной респираторной поддержкой, способствующей улучшению параметров газового гомеостаза в группах. Соответственно, более ранняя активизация пациентов и восстановление перистальтики кишечника и функции ЖКТ в раннем послеоперацион- 


\begin{tabular}{|lcc|}
\hline Таблица 2. Частота и структура послеоперационных \\
критических инцидентов в исследуемых группах
\end{tabular}

P-достоверность различий между 1-й и 2-й группой;

$\chi^{2}$ - значение критерия Пирсона между 1-й и 2-й группой; жирным шрифтом выделена разница между группами при $p<0,05$.

ном периоде в 1-й группе способствовали меньшему времени пребывания пациентов в отделении реанимации и сокращению срока госпитализации в целом у пациентов 1-й группы, чему в немалой степени способствовал продленный симпатический эпидуральный блок [13]. Анализ эффективности послеоперационной анальгезии показал достижение более полноценного обезболивания у пациентов 1-й группы на 2-е сутки послеоперационного периода, что объясняется, вероятно, окончанием действия ТАР-блока. В то же время следует отметить, что уровень болевой импульсации в раннем послеоперационном периоде в 1-е и 2-е сутки послеоперационного периода в 1-й и 2-й группах не превышал значения 35 мм по ВАШ. При этом оптимально допустимым уровнем боли в послеоперационном периоде принято считать сине-голубую зону, находящуюся в диапазоне от 0 до 40 мм по 100 миллиметровой ВАШ [14]. Таким образом, применение ПЭА и ТАР-блокады для послеоперационной анальгезии показало свою высокую эффективность и позволило снизить применение опиоидов в послеоперационном периоде. Это обусловило снижение частоты и риска развития осложнений, связанных с системным введением опиоидов и послеоперационной гипокинезии, улучшило качество послеоперационной реабилитации и, соответственно, уменьшило сроки госпитализации. Полученные результаты исследования свидетельствуют о том, что сочетанная анестезия с применением ПЭА оказывает наиболее благоприятное влияние на течение раннего послеоперационного периода за счет более раннего восстановления кишечника. Сочетанная анестезия с применением ТАР-блокады позволяет проводить ее на достаточном уровне глубины, поддерживать адекватную анальгезию в послеоперационном периоде, показывая сопоставимую эффективность послеоперационного обезболивания с ПЭА. Следовательно, ТАР тоже имеет свою нишу в практике анестезиологического обеспечения при симультанной гернио- и абдоменопластике больших ПВГ у пациентов с ожирением.

\section{Выводы}

1. Использование сочетанной анестезии на основе десфлурана с продленной эпидуральной анальгезий ропивакаином ассоциируется с меньшим количеством послеоперационных критических инцидентов, способствуя ранней активизации и сокращению сроков пребывания пациентов в стационаре.

2. Применение сочетанной анестезии с использованием ТАР-блокады является приемлемым альтернативным методом анестезиологического обеспечения симультанной гернио- и абдоменопластики больших ПВГ у пациентов с ожирением при наличии противопоказаний для выполнения центральных нейроаксиальных методов обезболивания или больших технических сложностях для их выполнения.

Конфликт интересов. Авторы заявляют об отсутствии конфликта интересов.

Вклад авторов. Неймарк М.И. - научное руководство, анализ результатов исследования, редактирование статьи; Киселев Р.В. - набор клинического материала, анализ результатов исследования, написание статьи.

\section{ORCID авторов}

Неймарк М.И. - 0000-0001-9135-6392

Киселев Р.В. - 0000-0002-4390-0610 


\section{Литература/References}

[1] Le Huu Nho R., Mege D., Ouaïssi M., Sielezneff I., Sastre B. Incidence and prevention of ventral incisional hernia. J. Visc. Surg. 2012; 149(5): 3-14. DOI: 10.1016/j.jviscsurg.2012.05.004

[2] Hernández-Granados P., López-Cano M., Morales-Conde S., et al. Incisional hernia prevention and use of mesh. A narrative review. Cir Esp. 2018; 96(2): 76-87. DOI: 10.1016/j.ciresp.2018.01.003

[3] Yamamoto M., Takakura Y., Ikeda S., et al. Visceral obesity is a significant risk factor for incisional hernia after laparoscopic colorectal surgery: A single-center review. Asian J. Endosc. Surg. 2018; 19. DOI: 10.1111/ases.12466

[4] Parés D., ShamaliA., Stefan S., et al. Predictive factors for extraction site hernia after laparoscopic right colectomy. Int. J. Colorectal Dis. 2016; 31(7): 1323-1328. DOI: 10.1007/s00384-016-2610-x

[5] Silecchia G., Campanile F.C., Sanchez L., et al. Laparoscopic ventral/ incisional hernia repair: updated Consensus Development Conference based guidelines [corrected].Surg Endosc. 2015; 29(9): 246384. DOI: $10.1007 / \mathrm{s} 00464-015-4293-8$

[6] Хашимов Б.Б., Аутлев К.М., Кручинин Е.В. и др. Частота возникновения грыж передней брюшной стенки у пациентов с морбидным ожирением. Уральский медицинский журнал. 2017; 3: 107-110.

[Hashimov B.B., Autlev K.M., Kruchinin E.V., et al. The incidence of hernia of the anterior abdominal wall in patients with morbid obesity. Ural'skij medicinskij zhurnal. 2017; 3: 107-110. (In Russ)]

[7] Hebbard P, Fujiwara Y, Shibata Y, Royse C. Ultrasound-guided transversus abdominis plane (TAP) block. Anaesthesia and Intensive Care 2007; 35: 616-617.

[8] Белоярцев, Ф.Ф. Компоненты общей анестезии. М.: Медицина, 1997.

[Beloyarcev F.F. Components of General Anesthesia. M.: Meditsina,1997.(In Russ)]

[9] Эпштейн СЛ. Периоперационное анестезиологическое обеспечение больных с морбидным ожирением. Регионарная анестезия и лечение острой боли. 2012; 4(3): 5-27.
[Ehpshtejn S.L. Perioperative anesthetic management of patients with morbid obesity. Regionarnaya anesteziya i lechenie ostroj boli. 2012; 4(3): 5-27.(In Russ)]

[10] Gaston-Johanson F., Albert M., Fagan E., Zimmerman L. Similarities in pain description of four different ethnic-culture groups. J.Pain Symptom Manage.1990; 5(2): 94-100. DOI: //doi.org/10.1016/ S0885-3924(05)80022-3

[11] Вейлер Р.В., Мусаева Т.С., Трембач Н.В., Заболотских И.Б. Критические инциденты в течение комбинированной анестезии при обширных абдоминальных операциях у пациентов пожилого и старческого возраста: роль предоперационного уровня бодрствования. Анестезиология и реаниматология. 2016; 61(5). DOI: http://dx.doi.org/10.18821/0201-7563-2016-61-5-352-356

[Vejler R.V., Musaeva T.S., Trembach N.V., Zabolotskih I.B. Critical incidents during combined anesthesia during extensive abdominal operations in patients of elderly and senile age: the role of the preoperative level of wakefulness. Anesteziologiya i reanimatologiya. 2016; 61(5). DOI: http://dx.doi.org/10.18821/02017563-2016-61-5-352-356. (In Russ)]

[12] Лихванцев В.В. Критические инциденты при современных методах общей анестезии. Клиническая анестезиология и реаниматология. 2007; (4): 42.

[Lihvancev V.V. Critical incidents with modern methods of general anesthesia. Klinicheskaya anesteziologiya i reanimatologiya. 2007; (4): 42 (In Russ)]

[13] Анисимов М.А., Горобец, Е.С., Якушина И.А. Эффективная анестезия при выполнении онкогинекологических операций у пациенток с сопутствующим морбидным ожирением. Вестник анестезиологии и реаниматологии. 2015; 6: 46-52.

[Anisimov M.A., Gorobec E.S., Yakushina I.A. Effective anesthesia when performing oncologic operations in patients with concomitant morbid obesity. Vestnik anesteziologii i reanimatologii. 2015; 6: 46-52. (In Russ)]

[14] Vadivelu N., Mitra S., Narayan D. Recent advances in postoperative pain management. Yale J. Biol. Med. 2010; 83(1): 11-25.

Поступила 29.09.2018 